\title{
AFP Gene Hepatocellular Carcinoma Vaccine
}

National Cancer Institute

\section{Source}

National Cancer Institute. AFP Gene Hepatocellular Carcinoma Vaccine. NCI Thesaurus.

Code C2512.

A cancer vaccine composed of naked plasmid DNA of the gene for the tumor-associated antigen alpha-fetoprotein (AFP), a macromolecule that acts as a specific immunologic target for hepatocellular carcinoma. This agent exerts an antitumor effect by inducing cytotoxic T-lymphocytes to attack AFP-expressing tumor cells. ( $\mathrm{NCI04)}$ 